\title{
Coherent Electromagnetic Processes in Ultra-Peripheral Heavy-Ion Collisions
}

\author{
F. Meissner and V.B. Morozov for the STAR Collaboration \\ Lawrence Berkeley National Laboratory, One Cyclotron Rd., Berkeley, CA 94720
}

\begin{abstract}
We report measurements for coherent $\rho^{0}$ production, $A u A u \rightarrow A u A u \rho^{0}$, and coherent $\rho^{0}$ and $e^{+} e^{-}$pair production accompanied by mutual nuclear Coulomb excitation, $A u A u \rightarrow A u^{\star} A u^{\star} \rho^{0}$ and $A u A u \rightarrow A u^{*} A u^{*}+e^{+} e^{-}$, in ultra-peripheral relativistic gold-gold collisions (UPC). We discuss transverse momentum, mass, and rapidity distributions. The two photon-process of $e^{+} e^{-}$pair production is an important probe of strong field QED because of the large coupling $Z \alpha=0.6$. At $\sqrt{s_{N N}}=200 \mathrm{GeV}$, the $e^{+} e^{-}$production cross section agrees with lowest order QED calculations. The cross sections for coherent $\rho^{0}$ production at $\sqrt{s_{N N}}=130$ and $200 \mathrm{GeV}$ are in agreement with theoretical predictions. The calculations for both, coherent $e^{+} e^{-}$and $\rho^{0}$ production treat nuclear excitation as independent process.
\end{abstract}

In ultra-peripheral heavy ion collisions, photon exchange, photon-photon or photon-nuclear interactions take place at impact parameters $b$ larger than twice the nuclear radius $R_{A}$, where no nucleon-nucleon collisions occur [1]. Examples are nuclear Coulomb excitation, electron-positron pair and meson production, and vector meson production. The exchange bosons can couple coherently to the nuclei, yielding large cross sections. Coherence restricts the final states to low transverse momenta, a distinctive experimental signature. We report measurements for coherent $e^{+} e^{-}$pair and $\rho^{0}$ production, where both processes may be accompanied by mutual nuclear Coulomb excitation, $A u A u \rightarrow A u^{(\star)} A u^{(\star)} e^{+} e^{-}$ and $A u A u \rightarrow A u^{(\star)} A u^{(\star)} \rho^{0}$.

The purely electromagnetic process of $e^{+} e^{-}$ pair production is shown in lowest order in Fig. 11. The photon flux emitted by the gold ions can be described by the Weizsäcker-Williams approach [2]. The $e^{+} e^{-}$pair production is then a result of a two-photon collision. The gold nuclei are not disrupted, and the final state consists solely of two nuclei and two leptons.

The quasi-real photons couple coherently to the $\mathrm{Au}$ ions; the photon flux of a single ion is proportional to the square of the nuclear charge $Z^{2}$ and the cross-section scales as $Z^{4}$. The total cross section for $e^{+} e^{-}$pair production in ultra-peripheral gold-gold collisions at $\sqrt{s_{N N}}=200 \mathrm{GeV}$ is $33 \mathrm{~kb}$ in lowest-order QED. The applicability of QED perturbation theory for $e^{+} e^{-}$pair production is questionable since the photon coupling is large, $Z \alpha \sim 0.6$, and this process becomes an important test of strong field QED [1].

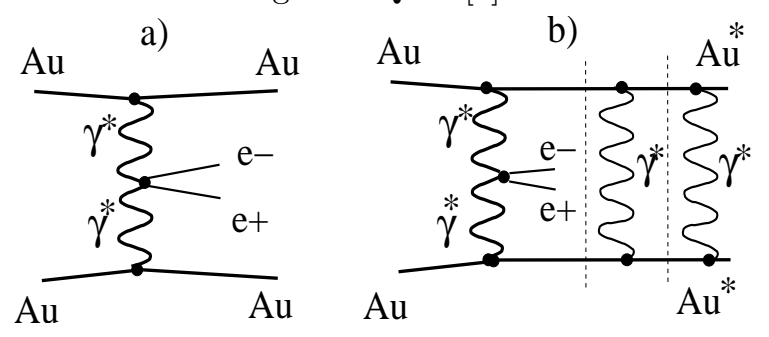

Figure 1. Diagram for (a) exclusive $e^{+} e^{-}$pair production in ultra-peripheral heavy ion collisions, and (b) pair production with nuclear excitation. The dashed lines indicate factorization.

In addition to coherent $e^{+} e^{-}$production, the exchange of virtual photons may excite the nuclei (Fig. 1b). In lowest order, mutual nuclear excitation of heavy ions occurs by the exchange of two photons 34. Pair production and nuclear excitation factorize for heavy-ion collisions, since non-factorisable diagrams are small [5]. Because of the Coulomb barrier for the emission of charged particles, nearly all nuclear decays following photon absorption include neutron emission [6]. 
Exclusive $\rho^{0}$ meson production, $A u A u \rightarrow$ $A u A u \rho^{0}$ (Fig. 2a), can be described by the vector meson dominance model 77. A photon emitted by one nucleus fluctuates to a virtual $\rho^{0}$ meson, which scatters elastically from the other nucleus, yielding a final state of two nuclei and the vector meson decay products $[8]$. The additional exchange of virtual photons may excite the nuclei (Fig. 2b) yielding subsequent neutron emission. Justified by the similar case of two-photon interactions, both processes are assumed to factorize. Calculations for both, coherent $e^{+} e^{-}$and $\rho^{0}$ production with nuclear excitation assume that these processes are independent, sharing only a common impact parameter 83.

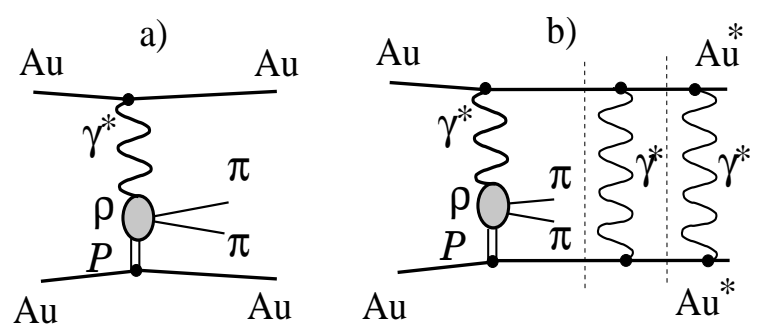

Figure 2. Diagram for (a) exclusive $\rho^{0}$ production in ultra-peripheral heavy ion collisions, and (b) $\rho^{0}$ production with nuclear excitation. The dashed lines indicate factorization.

In the rest frame of the target nucleus, midrapidity $\rho^{0}$ production at RHIC corresponds to a photon energy of $50 \mathrm{GeV}$ and a photon-nucleon center-of-mass energy of $10 \mathrm{GeV}$. At this energy, Pomeron $(\mathcal{P})$ exchange dominates over meson exchange, as indicated by the rise of the $\rho^{0}$ production cross section with increasing energy in lepton-nucleon scattering. The $\rho^{0}$ production cross sections are large: the photon flux is proportional to $Z^{2}$ [2], and the forward cross section for elastic $\rho^{0} A$ scattering $d \sigma^{\rho A} /\left.d t\right|_{t=0}$ scales as $A^{4 / 3}$ for surface coupling and $A^{2}$ in the bulk limit. At a center-of-mass energy of $\sqrt{s_{N N}}=130(200) \mathrm{GeV}$, a total $\rho^{0}$ cross section, regardless of nuclear excitation, $\sigma\left(A u A u \rightarrow A u^{(\star)} A u^{(\star)} \rho^{0}\right)=350(590) \mathrm{mb}$ is predicted from a Glauber extrapolation of $\gamma p \rightarrow \rho^{0} p$ data 8 .

The coherent coupling of photon and Pomeron to the nuclei yields a unique experimental signature: the central system is created a low transverse momentum. For $e^{+} e^{-}$pair production, the pair- $p_{T}^{e e} \sim 2 M_{e e} / \gamma$ is only a few $\mathrm{MeV}$ in our accessible kinematic. For $\rho^{0}$ production, the wavelength $\lambda_{\gamma, \mathcal{P}}>2 R_{A}$ leads to the coherence condition of $p_{T}<\pi \hbar / R_{A}(\sim 90 \mathrm{MeV} / \mathrm{c}$ for gold with $\left.R_{A} \sim 7 \mathrm{fm}\right)$ and a maximum longitudinal momentum of $p_{\|}<\pi \hbar \gamma / R_{A}(\sim 6(9) \mathrm{GeV} / \mathrm{c}$ at $\gamma=70(108))$, where $\gamma$ is the Lorentz boost of the nucleus.

In the years 2000 and 2001, RHIC collided gold nuclei at a center-of-mass energy of $\sqrt{s_{N N}}=130$ and $200 \mathrm{GeV}$, respectively. The STAR detector consists of a $4.2 \mathrm{~m}$ long cylindrical time projection chamber (TPC) of $2 \mathrm{~m}$ radius. In 2000 the $\mathrm{TPC}$ was operated in a $0.25 \mathrm{~T}$ solenoidal magnetic field. In 2001 the magnetic field was at design value of $0.5 \mathrm{~T}$ with a small data set taken at $0.25 \mathrm{~T}$. Particles are identified by their energy loss in the TPC. A central trigger barrel (CTB) of 240 scintillator slats surrounds the TPC. Two zero degree calorimeters (ZDC) at $\pm 18 \mathrm{~m}$ from the interaction point are sensitive to the neutral remnants of nuclear break-up.

$A u A u \rightarrow A u^{\star} A u^{\star} e^{+} e^{-}$

Exclusive $e^{+} e^{-}$pair production in UPC has a distinctive experimental signature: the lepton pair is observed in an otherwise 'empty' spectrometer. The tracks are approximately backto-back in the transverse plane due to the small $p_{T}$ of the pair. For the analysis of $A u A u \rightarrow$ $A u^{\star} A u^{\star} e^{+} e^{-}$we use a 800.000 event sample at $\sqrt{s_{N N}}=200 \mathrm{GeV}$ with the $0.25 \mathrm{~T}$ magnetic field setting. These events were selected by the 'minimum bias trigger' which requires coincident neutron signals in the East and West ZDCs, i.e. mutual nuclear excitation.

We select events with two oppositely charged tracks, emerging from the interaction region. At momenta below $125 \mathrm{MeV} / \mathrm{c}, e^{+} e^{-}$pairs are identified by their energy loss in the TPC as shown 
in Fig. 3. To reconstruct a track with at least 90\% efficiency requires a minimum track momentum at mid-rapidity $\left(\left|\eta^{\text {track }}\right|<1.15\right)$ of $p_{T}^{\text {track }}>$ $65 \mathrm{MeV} / \mathrm{c}$ at $0.25 \mathrm{~T}$. These requirements limit the observed $e^{+} e^{-}$pairs to the kinematic range of pair invariant mass $130 \mathrm{MeV}<W_{e^{+} e^{-}}<265 \mathrm{MeV}$, pair rapidity $|Y|<1.15$ and $\cos \left(\theta^{*}\right)<0.7$. Here, $\theta^{\star}$ is the angle between $e^{+}$and the photon momentum in the $\gamma \gamma$ center-of-mass frame (approximately the beam axis).

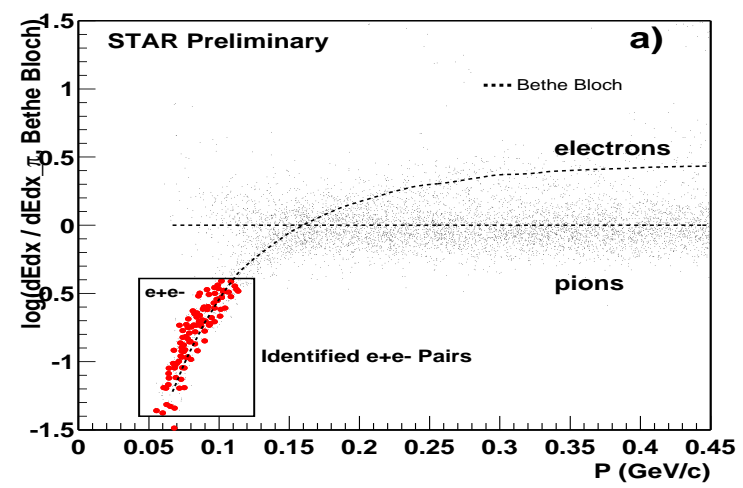

Figure 3. Energy loss $d E / d x$ of tracks in the 2track, $\sqrt{s_{N N}}=200 \mathrm{GeV}$ minimum bias data with reduced magnetic field. The dots indicate events where both particles are identified as electrons.

We have identified $61 e^{+} e^{-}$ultra-peripheral pairs in the event sample collected with the minimum bias trigger at $0.25 \mathrm{~T}$. The efficiency corrected $p_{T}^{e e}$ spectrum in Figure 4 (dots) shows a clear peak at $p_{T}<20 \mathrm{MeV} / \mathrm{c}$ identifying the process $A u A u \rightarrow A u^{\star} A u^{\star} e^{+} e^{-}$.

We have developed a Monte Carlo simulation of the $e^{+} e^{-}$pair production with mutual nuclear excitation 910. The photon fluxes from the $\mathrm{Au}$ ions are calculated according to the WeizsäckerWilliams equivalent photon approximation. The simulation uses the lowest-order QED approximation for the $\gamma \gamma \rightarrow e^{+} e^{-}$annihilation and assumes that the pair production is independent of electromagnetic excitation of the Au ions. Except for the lowest $p_{T}$ bin, the data in Figure 4 are in

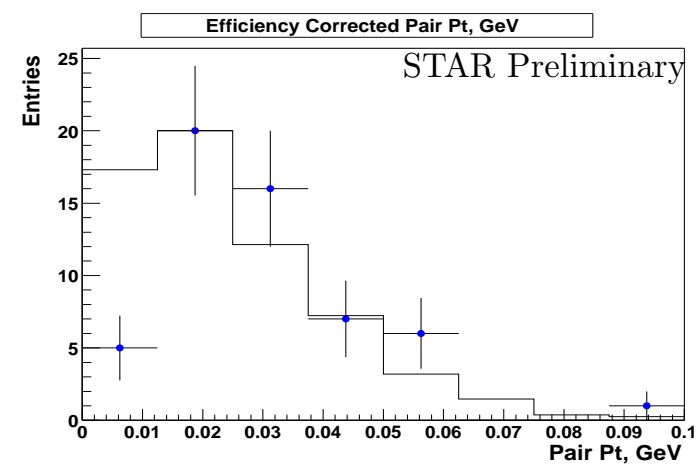

Figure 4. Efficiency corrected total transverse momentum spectrum of the identified $e^{+} e^{-}$pairs (dots) and scaled Monte Carlo prediction (solid).

good agreement with a normalized Monte Carlo simulation (histogram).

The integrated luminosity for the minimum bias data is determined from the number of hadronic interactions, assuming a total gold-gold hadronic cross section of $7.2 \mathrm{~b}[3$. The differential cross-section $d \sigma^{e e} / d W$ is presented in Figure [5] compared to Monte Carlo simulations. Within the limited kinematic range quoted above the integrated cross-section for coherent electron pair production was found to be $6.0 \mathrm{mb} \pm 17 \% \pm 19 \%$ which agrees well with the Monte Carlo prediction of $7.8 \mathrm{mb}$. The major contributions for the systematic uncertainty come from the luminosity determination, efficiency corrections for tracking and vertex finding, and acceptance correction.

The cross section $d \sigma\left(A u A u \rightarrow e^{+} e^{-}\right) / d W_{\gamma \gamma}$ is maximal at low invariant mass $W_{\gamma \gamma} \sim 2 m_{e}$ and falls rapidly as $1 / W_{\gamma \gamma}^{4}$; the pair production is peaked at small forward angles $\theta^{\star} \sim 1 / \gamma$. Including the requirement of nuclear breakup, only a small $\left(10^{-6}\right)$ fraction of the total cross section for coherent lepton pair production is observable. Even at the $0.25 \mathrm{~T}$ field, low momentum electrons do not reach the central trigger barrel, and triggering is limited to the minimum bias trigger, i.e. $e^{+} e^{-}$pair production with mutual nuclear excitation. A larger kinematic acceptance for $e^{+} e^{-}$pair production could be accomplished by lowering the field of the STAR magnet. In the limited kinematic range and limited by the 


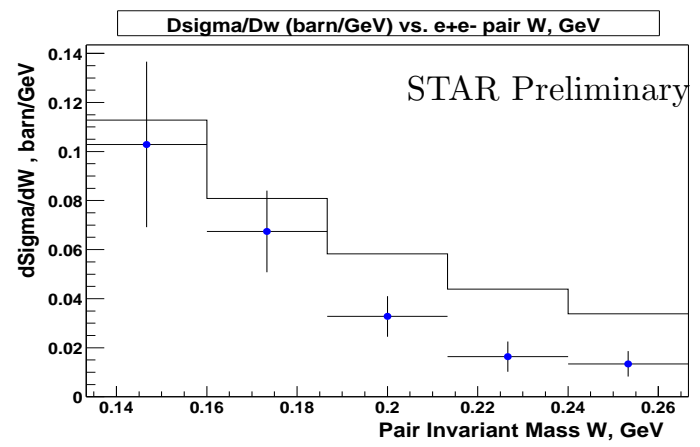

Figure 5. Differential cross-section vs. pair invariant mass for the data (dots) and Monte Carlo prediction (solid).

available statistics, our measurement agrees with leading order QED calculations. Extending these measurements to lower values of $W_{\gamma \gamma}$ would allow to access the kinematic region, where the contributions of higher order effects are expected to become sizable.

\section{$A u A u \rightarrow A u A u \rho^{0}$ and $A u A u \rightarrow A u^{\star} A u^{\star} \rho^{0}$}

Two different triggers are used for the $\rho^{0}$ analysis. For $A u A u \rightarrow A u A u \rho^{0}$, about 30,000 (2000) and $1.5 \mathrm{M}(2001)$ events were collected using a low-multiplicity 'topology' trigger. The CTB was divided in four azimuthal quadrants. Single hits were required in the opposite side quadrants; the top and bottom quadrants acted as vetoes to suppress cosmic rays. A fast on-line reconstruction removed events without reconstructible tracks from the data stream. To study $A u A u \rightarrow$ $A u^{\star} A u^{\star} \rho^{0}$, about $0.8 \mathrm{M}(2000)$ and $2.5 \mathrm{M}(2001)$ 'minimum bias' events, which required coincident detection of neutrons from nuclear break-up in both ZDCs as a trigger, are used for the analysis.

Events are selected with exactly two oppositely charged tracks forming a common vertex within the interaction region. The specific energy loss $d E / d x$ in the TPC shows that the event sample is dominated by pion pairs. In the topology triggered data sets, without the ZDC requirement, cosmic rays are a major background. They are removed by requiring that the two pion tracks have an opening angle of less than 3 radians. Figure 6 shows kinematic distributions for the selected 2track events in the $\sqrt{s_{N N}}=200 \mathrm{GeV}$ minimum bias data; these distributions are similar for the other data sets.

Figure 6a) shows the transverse momentum spectrum of oppositely charged pion-pairs (points). A clear peak, the signature for coherent coupling, can be observed at $p_{T}<150 \mathrm{MeV} / \mathrm{c}$. Those events are compatible with coherently produced $\rho^{0}$ candidates. A background model from like-sign combination pairs (shaded histogram), which is normalized to the signal at $p_{T}>250$ $\mathrm{MeV} / \mathrm{c}$, does not show such a peak. The open histogram is a Monte Carlo simulation [8] for coherent $\rho^{0}$ production accompanied by nuclear breakup superimposed onto the background. The $d N^{\rho} / d p_{T}$ (i.e. the $d N^{\rho} / d t \sim d N^{\rho} / d p_{T}^{2}$ ) spectrum reflects not only the nuclear form factor, but also the photon $p_{T}$ distribution and the interference of production amplitudes from both gold nuclei. The interference arises since both nuclei can be either the photon source or the scattering target 11. A detailed analysis of the $p_{T}$ distribution is in progress.

The rapidity distribution in Fig. 6b) is well described by the reconstructed events from the Monte Carlo simulation. The generated rapidity distribution is also shown. The acceptance for exclusive $\rho^{0}$ is about $40 \%$ at $\left|y_{\rho}\right|<1$. At $\left|y_{\rho}\right|>1$, the acceptance is small and this region is excluded from the analysis; the cross sections are extrapolated to the full $4 \pi$ acceptance with the Monte Carlo simulation. Using the energy deposits in the ZDCs (Fig. 6r), we select events with at least one neutron $(\mathrm{xn}, \mathrm{xn})$, exactly one neutron $(1 \mathrm{n}, 1 \mathrm{n})$, or no neutrons $(0 \mathrm{n}, 0 \mathrm{n})$ in each ZDC; the latter occurs only in the topology trigger.

Figure 6]) shows the $d \sigma / d M_{\pi \pi}$ spectrum for events with pair- $p_{T}<150 \mathrm{MeV} / \mathrm{c}$ (points). The fit (solid) is the sum of a relativistic BreitWigner for $\rho^{0}$ production and a Söding interference term for direct $\pi^{+} \pi^{-}$production [12] (both dashed). A second order polynomial (dashdotted) describes the combinatorial background (shaded histogram) from grazing nuclear collisions and incoherent photon-nucleon interactions. The $\rho^{0}$ mass and width are consistent with ac- 

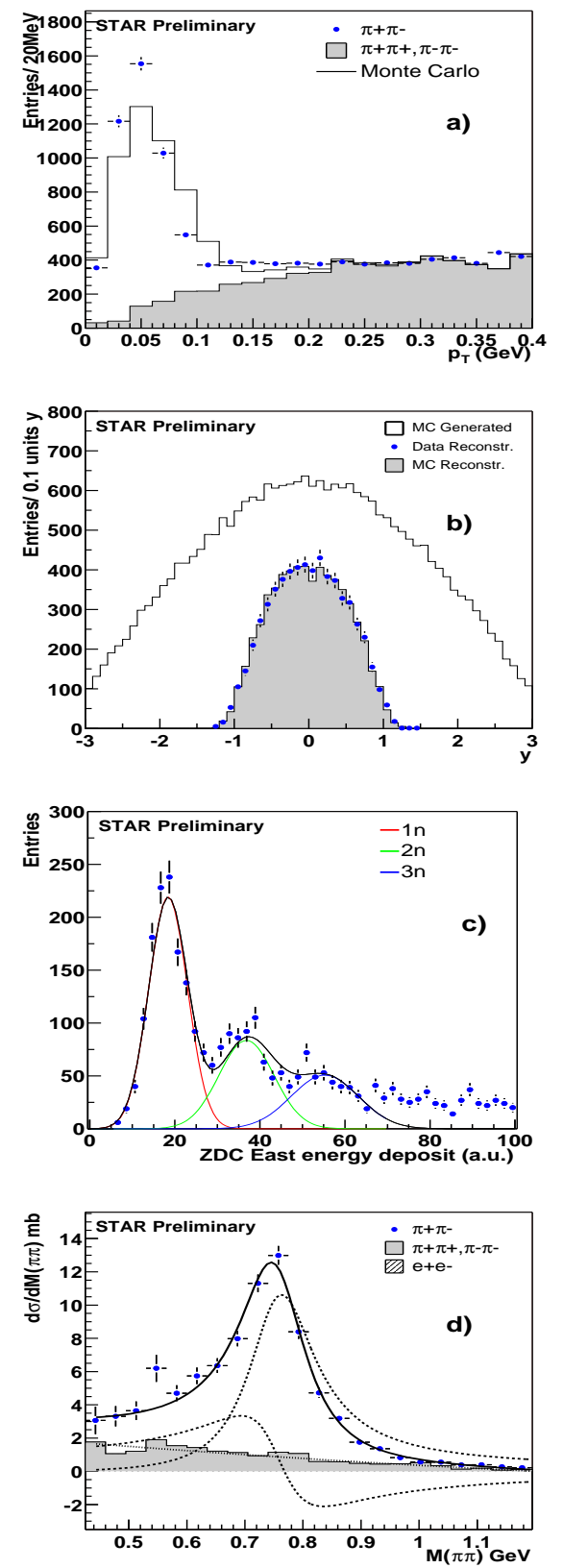

Figure 6. The (a) $\rho^{0}$ transverse momentum and (b) rapidity distribution, the (c) ZDC response, and (d) the $d \sigma / d M_{\pi \pi}$ invariant mass distribution for 2-track $(\mathrm{xn}, \mathrm{xn})$ events in the $\sqrt{s_{N N}}=200 \mathrm{GeV}$ minimum bias data. cepted values 13. Alternative parameterizations like a modified Söding parametrization 14 and a phenomenological Ross-Stodolsky parametrization 15 yield similar results. Incoherent $\rho^{0}$ production, where a photon interacts with a single nucleon, yields high $p_{T} \rho^{0} \mathrm{~s}$, which are suppressed by the low pair $p_{T}$ requirement; the remaining small contribution is indistinguishable from the coherent process. A coherently produced background arises from the two-photon process $A u A u \rightarrow A u^{(\star)} A u^{(\star)} l^{+} l^{-}$. It contributes mainly at low invariant mass $M_{\pi \pi}<0.5 \mathrm{GeV} / \mathrm{c}^{2}$. The small contribution from $\omega$ decays is neglected.

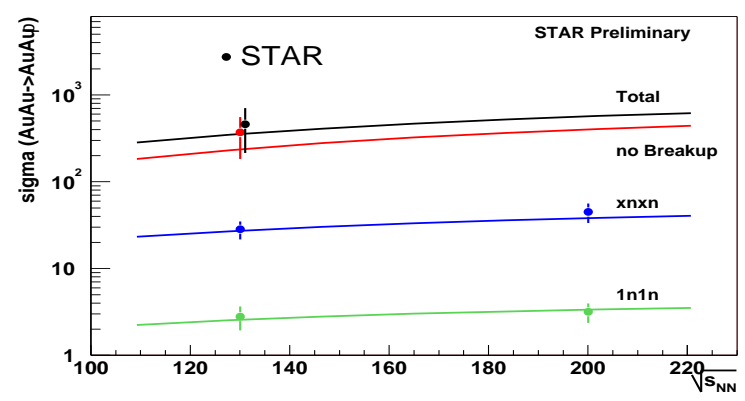

Figure 7. Comparison to predictions from Ref. [8].

Figure 7 compares our results on cross sections for coherent $\rho^{0}$ production at $\sqrt{s_{N N}}=$ $130 \mathrm{GeV}[16]$ to the calculations of Ref. [8]. Preliminary results for $\sqrt{s_{N N}}=200 \mathrm{GeV}$ are also shown in the plot. The cross sections are obtained from the integral of the Breit-Wigner fit, extrapolated to full rapidity. For coherent $\rho^{0}$ production accompanied by mutual nuclear break-up (xn,xn), we measure a cross section of $\sigma(A u A u \rightarrow$ $\left.A u_{x n}^{*} A u_{x n}^{*} \rho^{0}\right)=39.7 \pm 2.8 \pm 9.7 \mathrm{mb}$. By selecting single neutron signals in both ZDCs, we obtain $\sigma\left(A u A u \rightarrow A u_{1 n}^{*} A u_{1 n}^{*} \rho^{0}\right)=2.8 \pm 0.5 \pm 0.7 \mathrm{mb}$. The systematic uncertainties are dominated by the uncertainties of the luminosity determination and the $4 \pi$ extrapolation. The absolute efficiency of the topology trigger is poorly known and does not allow a direct cross section measurement. From $\sigma\left(A u A u \rightarrow A u_{x n}^{*} A u_{x n}^{*} \rho^{0}\right)$ and 


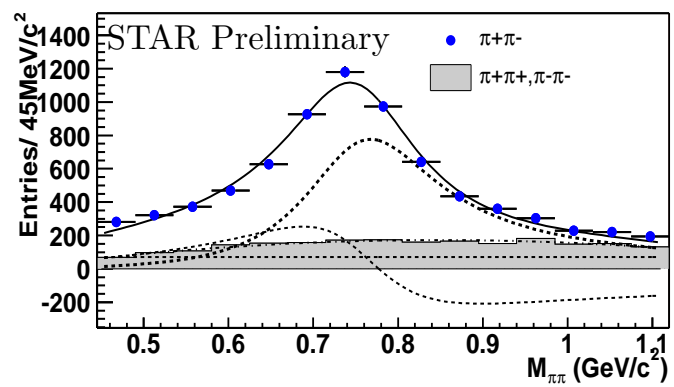

Figure 8. The $d N / d M_{\pi \pi}$ invariant mass distribution for 2-track events in $\sqrt{s_{N N}}=200 \mathrm{GeV}$ deuterium-gold collisions.

the ratio $\sigma_{x n, x n}^{\rho} / \sigma_{0 n, 0 n}^{\rho}$ we estimate $\sigma(A u A u \rightarrow$ $\left.A u A u \rho^{0}\right)=370 \pm 170 \pm 80 \mathrm{mb}$ and the total cross section for coherent $\rho^{0}$ production $\sigma(A u A u \rightarrow$ $\left.A u^{(*)} A u^{(*)} \rho^{0}\right)=460 \pm 220 \pm 110 \mathrm{mb}$. From a fit to the differential cross section $d \sigma(\gamma A u \rightarrow$ $\rho A u) / d t \sim d \sigma(\gamma A u \rightarrow \rho A u) / d p_{T}^{2} \propto e^{-b t}$ for the $(\mathrm{xn}, \mathrm{xn})$ events we obtain a forward cross section $d \sigma^{\rho A} /\left.d t\right|_{t=0}=965 \pm 140 \pm 230 \mathrm{mb} / \mathrm{GeV}^{2}$ and an approximate gold radius of $R_{A u}=\sqrt{4 b}=7.5 \pm 2 \mathrm{fm}$, comparable to previous results 17 .

In the year 2003, STAR recorded deuteriumgold $(\mathrm{dAu})$ collisions. In $d A u$ collisions two processes compete, where either gold or the deuterium are the photon emitter: $A u \rightarrow \gamma A u ; \gamma d \rightarrow$ $d \rho$ and $d \rightarrow \gamma d ; \gamma A u \rightarrow A u \rho$. About $1 \mathrm{M} d A u$ collisions where recorded using the low multiplicity topology trigger, with a sub-sample that included a requirement on a neutron signal in the ZDC, i.e. deuterium break up. Fig. [8 shows a clear $\rho^{0}$ signal in a preliminary $M \pi \pi$ invariant mass spectrum.

In summary, ultra-peripheral heavy-ion collisions are a new laboratory to study purely electromagnetic and diffractive interactions. The cross section for the two-photon process $A u A u \rightarrow$ $A u^{*} A u^{*}+e^{+} e^{-}$agrees with leading order QED calculations in the limited kinematic range presently experimentally accessible. The first measurements of coherent $\rho^{0}$ production with and without accompanying nuclear excitation, $A u A u \rightarrow A u^{\star} A u^{\star} \rho^{0}$ and $A u A u \rightarrow A u A u \rho^{0}$, con- firm the existence of vector meson production in ultra-peripheral heavy ion collisions. The cross sections at $\sqrt{s_{N N}}=130$ and $200 \mathrm{GeV}$ are in agreement with theoretical calculations. The calculations for both, coherent $e^{+} e^{-}$and $\rho^{0}$ production treat nuclear excitation as independent process.

\section{REFERENCES}

1. G. Baur et. al, Phys. Rept. 364, 359 (2002).

2. C. F. v. Weizsäcker, Z. Phys. 88, 612 (1934); E.J. Williams, Phys. Rev. 45, 729 (1934).

3. A. J. Baltz, C. Chasman, and S. N. White, Nucl. Instrum. Meth. A417, 1 (1998).

4. M. Chiu et al., Phys. Rev. Lett. 89, 012302 (2002).

5. K. Hencken, D. Trautmann and G. Baur, Z. Phys. C68, 473 (1995).

6. B. L. Berman and S. C. Fultz, Rev. Mod. Phys. 47, 713 (1975).

7. J.J. Sakurai, Ann.Phys. 111 (1960); T.H. Bauer et al., Rev. Mod. Phys. 50261 (1978).

8. S. R. Klein and J. Nystrand, Phys. Rev. C 60, 014903 (1999); J. Nystrand, A. J. Baltz and S. R. Klein, Phys. Rev. Lett. 89, 012301 (2002).

9. S.Klein and E.Scannapieco, Two-photon physics with STAR, STAR Note 243..

10. V. Morozov, Ph.D. Thesis, UC Berkeley, (2003).

11. S. R. Klein and J. Nystrand, Phys. Rev. Lett. 84, 2330 (2000).

12. P. Söding, Phys. Lett. 19, 702 (1966);

13. D. E. Groom et al., Eur. Phys. J. C15, 1 (2000).

14. J. Breitweg et al., Eur. Phys. J C2, 247 (1998).

15. M. Ross and L. Stodolsky, Phys. Rev. 149, 1172 (1966).

16. C. Adler et al., Phys. Rev. Lett. 89, 272302 (2002).

17. F. Bulos et al., Phys. Rev. Lett. 10, 490 (1969); H. Alvensleben et al., Phys. Rev. Lett. 24, 786 and 792 (1970), and Nucl. Phys. B18, 333 (1970); G. McClellan et al., Phys. Rev. D4, 2683 (1971). 